\title{
A Binarization Method for a Scenery Image with the Fractal Dimension
}

\author{
Hiromi Yoshida* and Naoki Tanaka \\ Kobe University \\ Graduate School of Maritime Science \\ 070w706w@stu.kobe-u.ac.jp, \\ ntanaka@maritime.kobe-u.ac.jp
}

\begin{abstract}
We propose a new binarization method suited for character extraction from a sign board in a scenery image. The binarization is thought to be a significant step in character extraction in order to get high quality result. Character region of sigh board, however, has many variation and colors. In addition to it, if there exists high frequency texture region like a mountain or trees in the background, it can be a cause of difficulty to binarize an image. At the high frequency region, the binarized result is sensitive to the threshold change. On the other hand, a character region of sign board consists of solid area, that is, includes few high frequency regions, and has relatively high contrast. So the binarized result of character region is stabile against an interval of the threshold value. Focusing attention on this point, we propose a new method which obtains a threshold value based on the fractal dimension to evaluate both region's density and stability to threshold change. Through the proposed method, we can get a fine quality binarized images, where the characters can be extracted correctly.
\end{abstract}

Keywords: Binarization Fractal dimension Blanket method.

\section{Introduction}

Binarization of gray level image is a significant step in region extraction and a number of binarization methods have been proposed. Trier[1] evaluated 15 binarization methods as promising by the procedure called goal-directed evaluation, and showed that Niblack's method[2] has the best performance as a local adaptive method and Otsu method[3] is the best in global methods. In these 15 methods, threshold value is selected based on the local or global statistical information of the gray level such as gray level histogram. Scenery image which contains sign board consists of many regions such as high or low texture, solid area, and have a contrast perturbation. In an image which has much color variation, the binarization methods which use gray level information only can generate poor results. So we introduce evaluating method of a binarized image using fractal dimension. The FD has relatively large peaks for texture regions and a stable interval for a character string region, i.e. sign board, respectively.

\footnotetext{
^ Corresponding author.
} 
By detecting the stable interval of a FD, we can obtain a threshold value which is the best value to obtain a fine binarized character string. That the fractal dimension can evaluate the fineness of a binarized result is reported by Yoshida[4]. The method detects cracks from considerably noisy road surface images.

The proposed method corresponds to a preprocessing step of character extraction method in a scenery image which contains a sign board. The proposed new binarization method uses a fractal dimension for evaluating binarized image to find a threshold value. By using our method, we can evaluate the density of regions and stability to threshold change, that is, connected components which are close to each other and consist of sold areas in input images. First, about fractal dimension is described. Then, we show algorithm of our technique and its experimental result.

\section{Associated Technique}

Fractal dimension was proposed as a method of texture analysis named "Blanket method" by SHUMEL[5] in 1984. The range of the dimension is from 2 to 3 and is obtained from expression $1,2,3$ and 4 .

$$
\begin{gathered}
U_{\epsilon}=\max \left\{U_{\epsilon-1}(i, j)+1, \max _{|(m, n)-(i, j)| \leq 1} U_{\epsilon-1}(m, n)\right\} \\
b_{\epsilon}=\min \left\{b_{\epsilon-1}(i, j)-1, \min _{|(m, n)-(i, j)| \leq 1} b_{\epsilon-1}(m, n)\right\} \\
A(\epsilon)=\frac{\sum_{i, j}\left(U_{\epsilon}(i, j)-b_{\epsilon}(i, j)\right)}{2 \epsilon} \\
A(\epsilon)=F \epsilon^{2-D}
\end{gathered}
$$

where $\epsilon$ is the number of blanket. Fractal dimension is calculated globally and also locally with a window. Noviant[6] proposed optimal range of the fractal dimension and adapts it locally to image and get a local fractal dimension (LFD) image. LFD image has a feature that brightness of region is proportional with frequency of texture region. Example of LFD images are shown at Fig.1. Window size for LFD is $3 \times 3$ and the blanket number is 44 .

\section{Binarization Algorithm}

Proposed algorithm has 5 step procedures as follows.

- Step1: Binarize the input image $\boldsymbol{I}(\boldsymbol{x})$ with every threshold values from $\mathbf{0}$ to $\mathbf{2 5 5}$, and obtained the 256 binarized images $\boldsymbol{I}_{\boldsymbol{b}_{\boldsymbol{i}}}(\boldsymbol{x})(\boldsymbol{i}=\mathbf{0}, \mathbf{1}, \ldots, \mathbf{2 5 5})$ respectively.

- Step2: The $\boldsymbol{F} \boldsymbol{D}(\boldsymbol{i})$ values can be calculated on $\boldsymbol{I}_{\boldsymbol{b}_{\boldsymbol{i}}}(\boldsymbol{x})$ images by the Blanket method. 


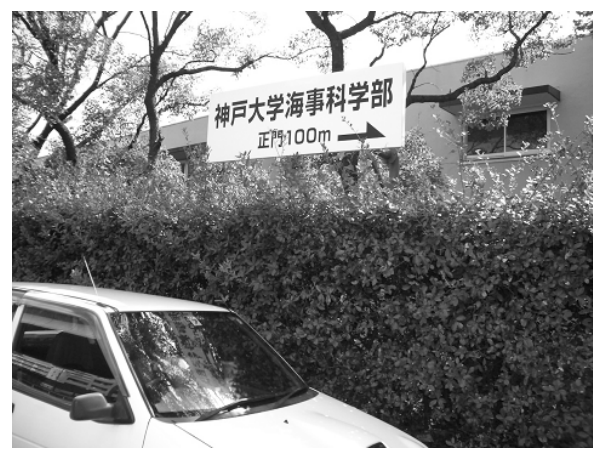

(a) Input image

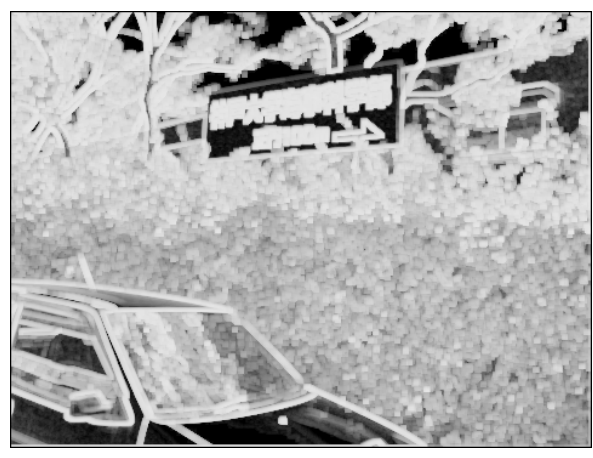

(b) LFD image

Fig. 1. Example of LFD image

- Step3: We treat the $\boldsymbol{F} \boldsymbol{D}(\boldsymbol{i})$ to be a function respect to $\boldsymbol{i}$. Then, smooth the $\boldsymbol{F} \boldsymbol{D}(\boldsymbol{i})$ to remove noise of the function, and find the stable interval of $\boldsymbol{F} \boldsymbol{D}(i)$ by taking the first derivation.

- Step4: In a stable interval, detect the minimum $\boldsymbol{F} \boldsymbol{D}(\boldsymbol{i})$ (which is not smoothed) as the threshold value $\boldsymbol{\theta}$.

- Step5: Final binarization Image $\boldsymbol{B}(\boldsymbol{x})$ can be obtained using the threshold value $\boldsymbol{\theta}$.

In the step3, we define the stable interval as follows: differentiate the smoothed $\boldsymbol{F} \boldsymbol{D}(i)$ with respect to $\boldsymbol{i}$, and count the number of $\frac{\delta}{\delta i} \boldsymbol{F} \boldsymbol{D}(\boldsymbol{i})$ which is equal $\mathbf{0}$ or nearly equal to $\mathbf{0}$ until it turns larger than $\mathbf{0} . \frac{\delta}{\delta i} \boldsymbol{F} \boldsymbol{D}(i)$ is calculated from expression 5 .

$$
\frac{\delta}{\delta i} F D(i)=F D(i+1)-F D(i)
$$

The longest interval where $\frac{\delta}{\delta i} \boldsymbol{F} \boldsymbol{D}(\boldsymbol{i})$ is flat defined as the stable interval. Example of graph of $\boldsymbol{F} \boldsymbol{D}(\boldsymbol{i})$ is shown at Fig.2.

\section{Experiment}

\subsection{Experimental Parameter}

Table 1 shows the parameters used in the experiment.

Noviant showed that appropriate range of $\epsilon$ is from 34 to 53. So we selected 44 as median of the range. Since $\epsilon$ in $\boldsymbol{F} \boldsymbol{D}(\boldsymbol{i})$ relates to evaluating the density of a regions, the binarized results are gradually changing. Fig. 3 showed how changing the binarized results. In the implementation, we use a "double-precision floatingpoint data type" for $\boldsymbol{F} \boldsymbol{D}(\boldsymbol{i})$ to decide a threshold value $\boldsymbol{\theta}$ precisely. 


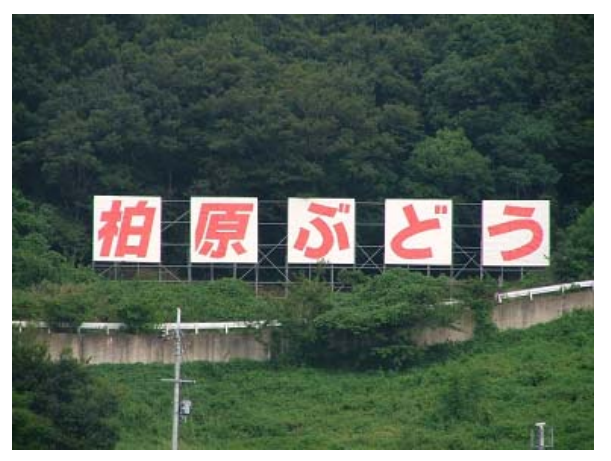

(a) Input image

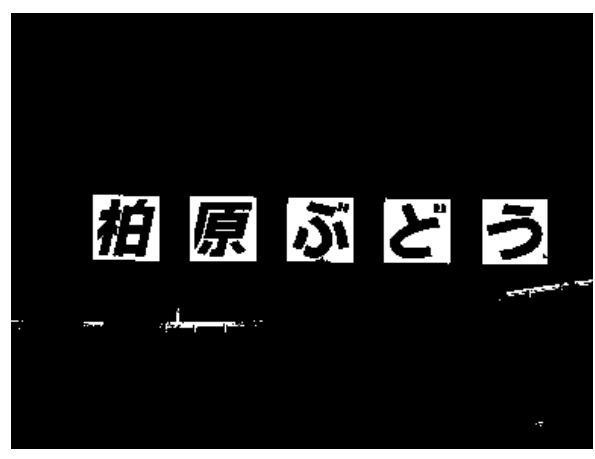

(b) Output image

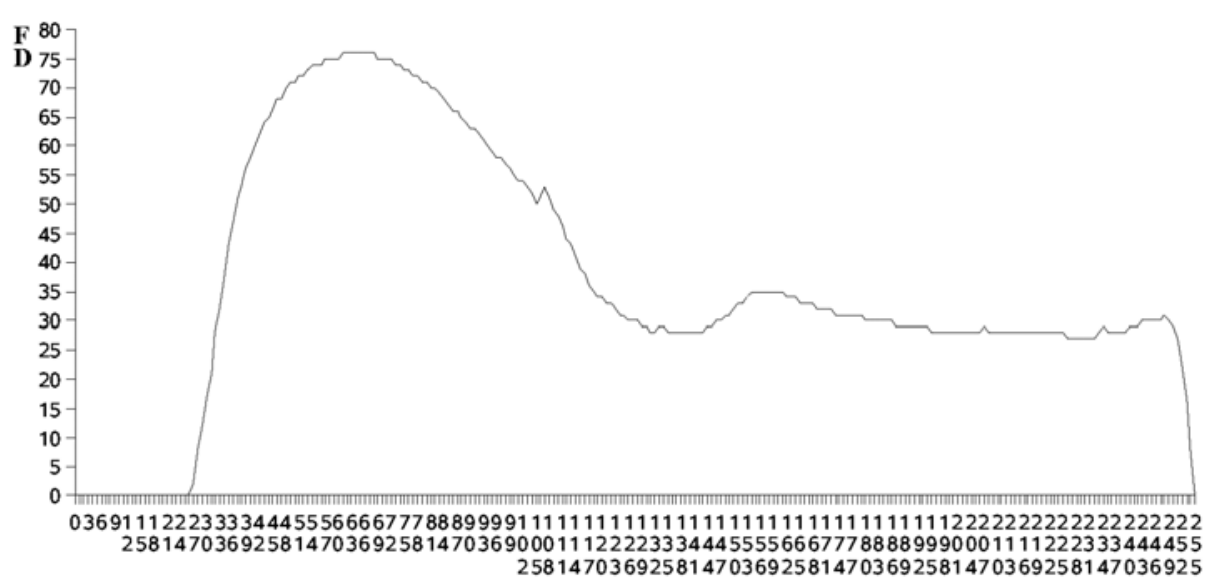

Threshold Value

(C) FD graph

Fig. 2. Graph of $\boldsymbol{F D}(\boldsymbol{i})$

Table 1. Experimental parameter

\begin{tabular}{c|cc}
\hline \hline & smoothed $\boldsymbol{F D ( i )}$ & $\boldsymbol{F D ( i )}$ \\
\hline$\epsilon$ & 44 & 44 \\
\hline Range of quantization & 100 & 100.0000 \\
\hline \hline
\end{tabular}




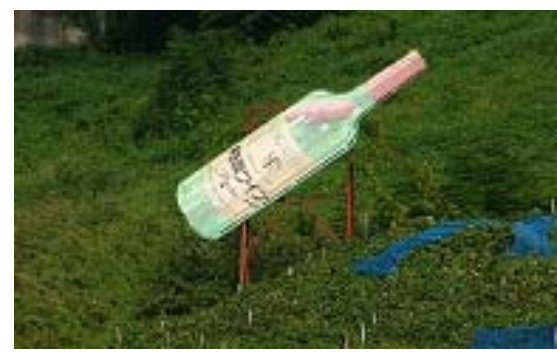

(a)Input image

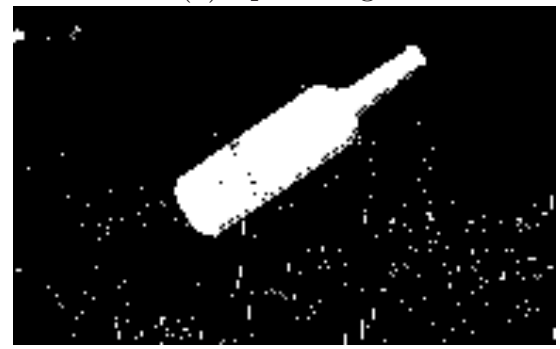

(c) $\epsilon=5$

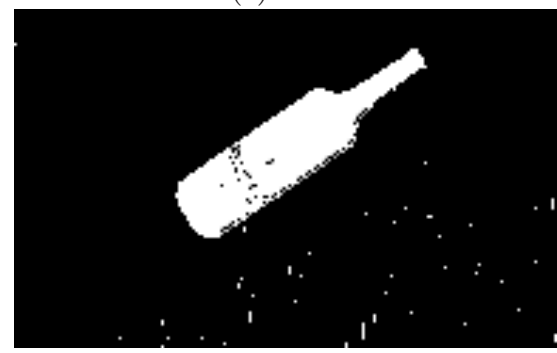

(e) $\epsilon=25$

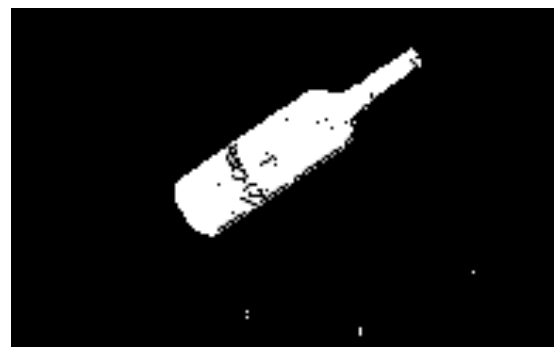

(b)Output image $(\epsilon=44)$

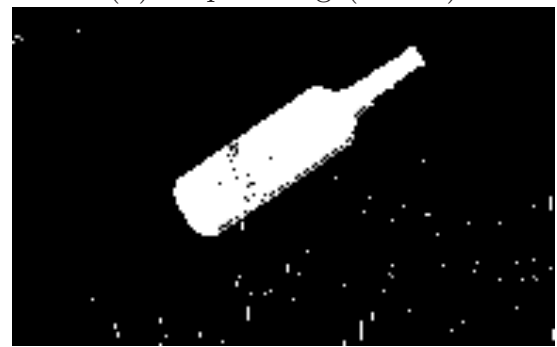

$(\mathrm{d}) \epsilon=10$

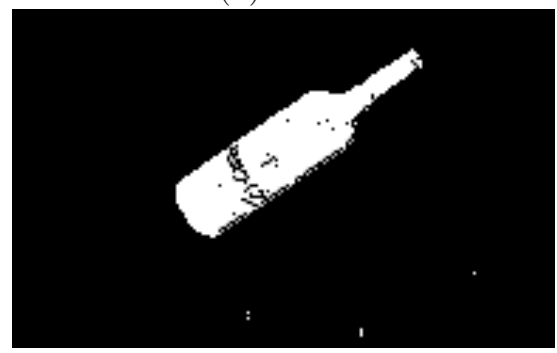

(f) $\epsilon=45$

Fig. 3. The examples of difference result due to change of blanket number

\subsection{Experimental Result}

Binarized images by proposed algorithm are shown at Fig.4. And processed results by Otsu's method and Niblack's method are also shown for comparison at Fig.5. We select these two methods because they have the best performance a promising binarization method using gray level information. The former is a local method; the latter is a global one. 


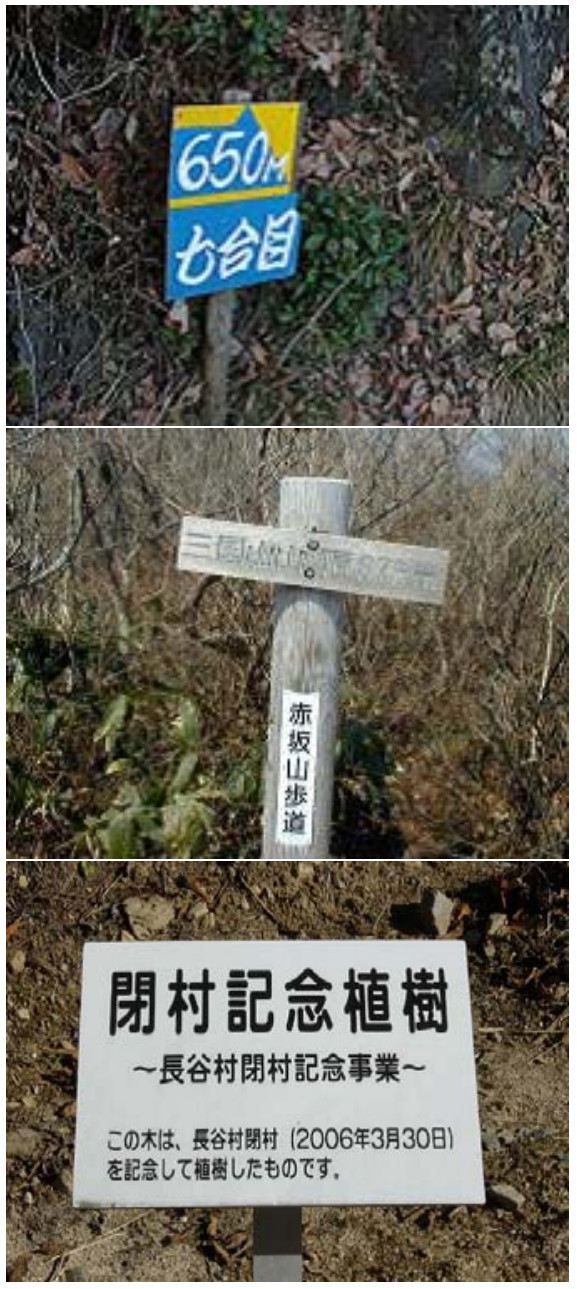

(a)Input image
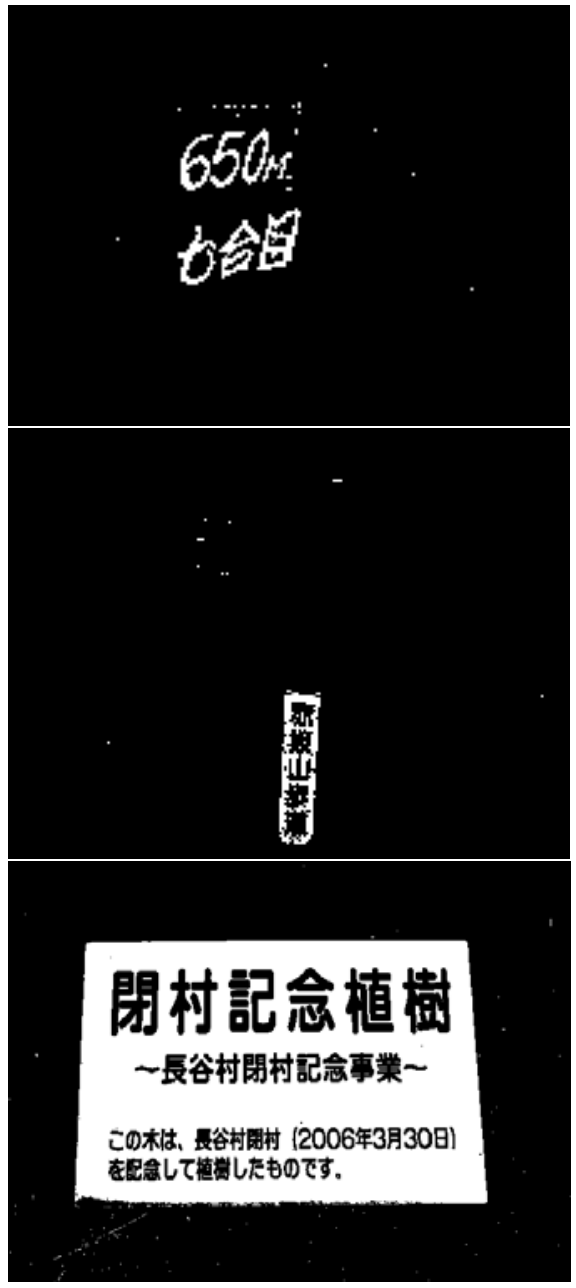

(b)Output image $(\epsilon=44)$

Fig. 4. The examples of binarized images by proposed method 


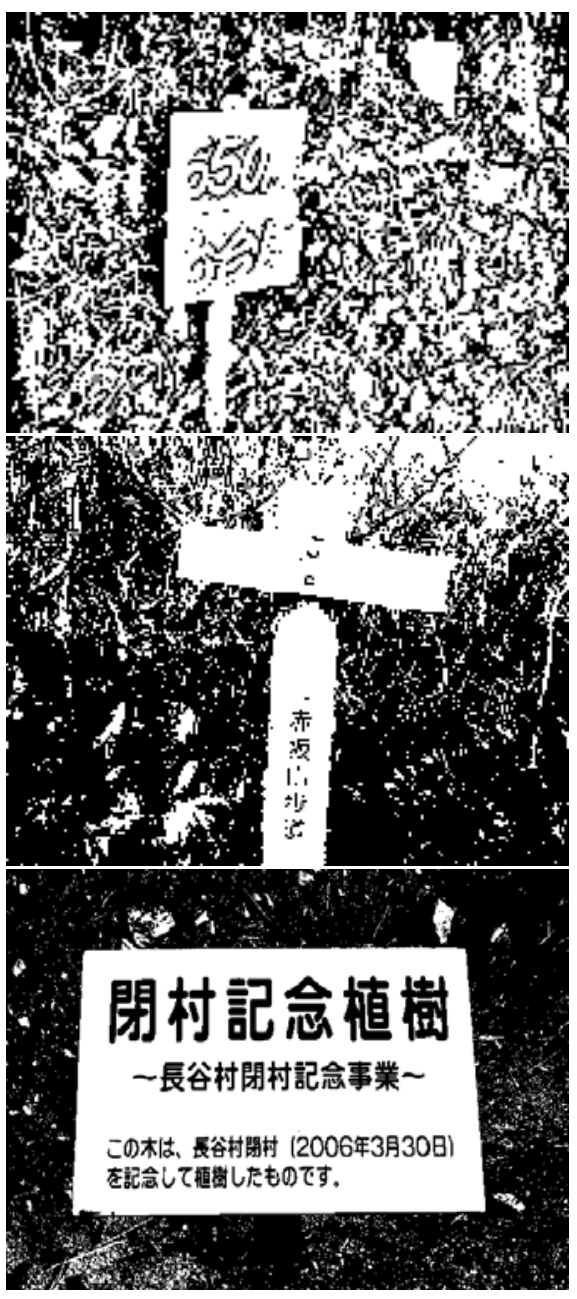

(a)Otsu's method

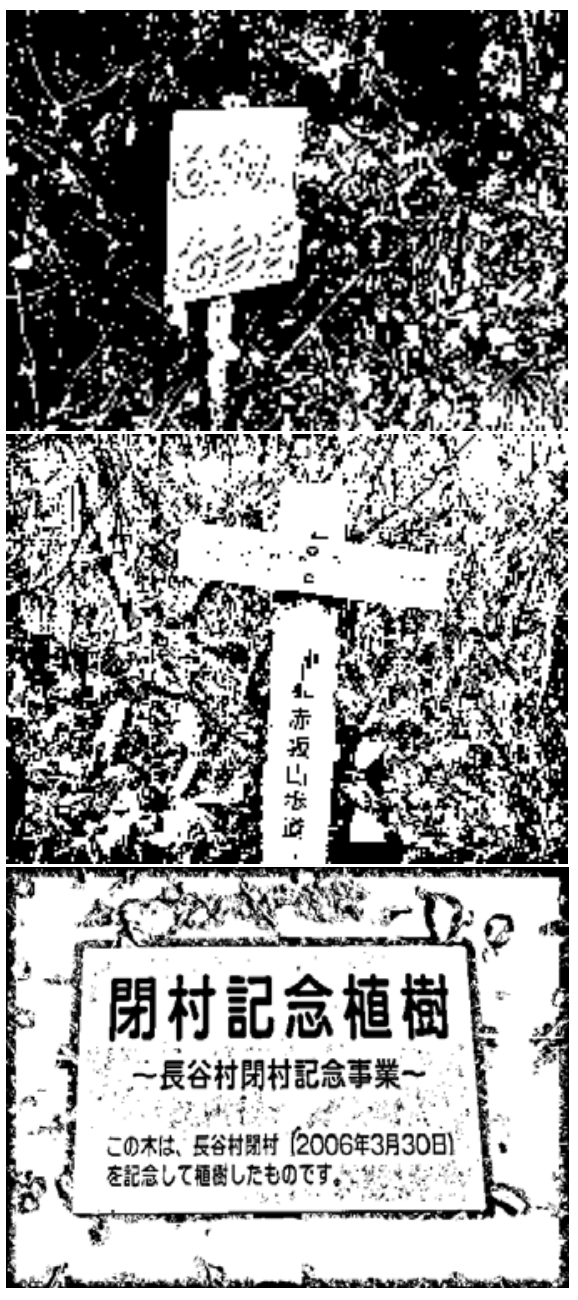

(b)Niblack's method

Fig. 5. The examples of binarized images by Otsu's method and Niblack's method

\section{Conclusion}

Result of experiment shows that the proposed method can be a promising binarization step of character detection method from sign board in a scenery image. Some noises are still remaining in binarized image. This is because our proposed method belongs to global techniques, that is, only one threshold value is applied for whole image. So we will develope a local adaptive binarization method based on this technique, and hope to get still improve the results. 


\section{References}

1. Trier, O.D., Jain, A.K.: Goal-Directed Evaluation of Binarization Methods. IEEE Trans. Pattern Analysis and Machine Inteligence 7(12), 1191-1201 (1995)

2. Niblack, W.: An Introduction to Digital Image Processing, pp. 115-116. Prentice Hall, Englewood Cliffs (1986)

3. Otsu, N.: A threshold selection method from gray-level histograms. IEEE Trans, Systems, Man, and Cybernetics 9(1), 62-66 (1979)

4. Yoshida, H., Tanaka, N.: A Binarization method for Crack Detection in a Road Surface Image with the Fractal Dimension. In: Proceedings of MVA 2009 IAPR Conference on Machine Vision Applications, pp. 70-73 (2009)

5. Peleg, S., Naor, J., Hartley, R., Avnir, D.: Multiple resolution texture analysis and classification. IEEE Trans. Pattern Analysis and Machine Inteligence 6(4), 518-523 (1984)

6. Noviant, S., Suzuki, Y., Maeda, J.: Optimumestimation of local fractal dimension based on the blanket method. IPSJ Journal 43(3), 825-828 (2002)

7. Wang, Y., Tanaka, N.: Text String Extraction from Scene Image Based on Edge Feature and Morphology. In: Proceedings of the 8th IAPR International Workshop on Document Analysis Systems, pp. 323-328 (2008)

8. Yoshida, H., Tanaka, N.: A Study on Signboard Image Identification with SIFT Features. In: Handout of 8th IAPR International Workshop on Document Analysis Systems, pp. 10-14 (2008) 